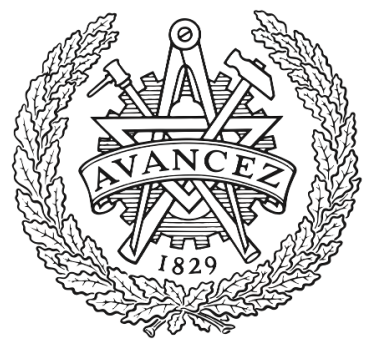

CHALMERS

UNIVERSITY OF TECHNOLOGY

\title{
Probabilistic spectrum Gaussian noise estimate for random bandwidth traffic
}

Downloaded from: https://research.chalmers.se, 2023-04-26 08:30 UTC

Citation for the original published paper (version of record):

Xu, Y., Agrell, E., Brandt Pearce, M. (2019). Probabilistic spectrum Gaussian noise estimate for random bandwidth traffic. IET Conference Publications. http://dx.doi.org/10.1049/cp.2019.0895

N.B. When citing this work, cite the original published paper. 


\title{
Probabilistic Spectrum Gaussian Noise Estimate for Random Bandwidth Traffic
}

\author{
Yuxin Xu ${ }^{11}$, Erik Agrell ${ }^{2}$ and Maite Brandt-Pearce ${ }^{1}$ \\ ${ }^{1}$ Department of Electrical and Computer Engineering, University of Virginia, USA \\ ${ }^{2}$ Department of Electrical Engineering, Chalmers University of Technology, Sweden \\ yx4vf@virginia.edu,mb-p@virginia.edu
}

Keywords: Elastic optical networks, random bandwidth traffic, physical layer impairments, Gaussian noise model

\begin{abstract} noise model applied to the maximum bandwidth.

\section{Introduction}

Elastic optical networks (EONs) have been proposed as an efficient solution to accommodate traffic demands for future communication needs [1]. Signals transmitted in long-haul fiber-optic networks suffer from interference and noise that impair the quality of transmission. Estimating these physical layer impairments (PLIs) is important in network planning of EONs and for allocating network resources. The Gaussian noise (GN) model was recently proposed to produce an accurate PLI estimate. The GN model is state dependent (taking the network state into account), instead of the oftenemployed worst-case PLI estimate provided by the transmission reach model. In this paper, we propose an alternative PLI model based on the GN model to account for randomly time-varying bandwidth traffic.
\end{abstract}

A probabilistic spectrum Gaussian noise (PSGN) model is proposed to predict the nonlinear noise for random bandwidth traffic in long-haul elastic optical networks. The model reduces the noise estimate $9.1 \%$ on average compared to the standard Gaussian

The GN model estimates the self-interference caused by the channel of interest and the cross-channel interference caused by signals transmitted on other channels on the same fiber link [2-5]. However, the GN model assumes that demands have a fixed bandwidth; when the given demands have time-varying bandwidth (random bandwidth), the GN model is not applicable. In state-of-the art techniques, time-varying demands are configured according to the maximum bandwidth envisioned, referred to as standard provisioning. For standard provisioning, noise is calculated based on the GN model using the largest foreseen bandwidth, which we refer to as the maximum bandwidth GN model. Unlike the TR model, this model is still network state dependent, but lacks awareness of the stochastic bandwidth. Standard provisioning using the maximum bandwidth GN PLI estimate leads to resource overprovisioning since the actual demands are time-varying with a predictable probability distribution. Our algorithm, named the probabilistic spectrum GN (PSGN) model, provides an accurate nonlinear noise estimate so that the quality of transmission can be guaranteed in the network planning process.

Researchers have recently proposed a statistical network assignment process (SNAP) algorithm based on Monte Carlo simulations to estimate the PLIs of random bandwidth demands [6,7]. Random data-rate demands are used in simulations to obtain expected network states and then calculate the average noise by utilizing the GN model over many trials. However, the SNAP algorithm is time consuming and computationally intense. For network planning with strict time constraints or with complicated resource allocation schemes, the SNAP algorithm is not able to provide the desired results.

The proposed PSGN model can be used to compute the expected noise as well as its variance, which can be used to generate a conservative estimate of the PLIs. It also provides simple closed-form expressions of the expected value and variance of the self-channel interference (SCI) and the expected value of cross-channel interference (XCI) when the probability density function (PDF) of the bandwidth of traffic demands is given and the spectrum assignment consists of defining a center frequency for each signal. The PSGN can be applied to any resource allocation schemes and requires hardly any computational resources. We show that the PSGN results in a $9.1 \%$ lower estimate of the nonlinear interference than the maximum bandwidth GN with an outage probability of less than $1.4 \%$.

\section{Traffic Model with Random Bandwidth Demands}

For each demand $q$ sharing the same link with the channel of interest, the bandwidth probability density function (PDF) and cumulative density function (CDF) corresponding to demand $q$ are assumed given and denoted as $f_{\Delta}^{(q)}(\delta)$, and $P_{\Delta}^{(q)}(\delta)$, respectively, where $\Delta$ is the random variable describing the bandwidth of demand $q$ with realization $\delta \in\left[\delta_{\min }, \delta_{\max }\right]$. The probability that a frequency $f$ is occupied by demand $q$ is denoted as $S^{(q)}(f)$. Let $I^{(q)}(f) \in\{0,1\}$ indicate the presence of traffic demand $q$ at frequency $f$; then $\operatorname{Pr}\left[I^{(q)}(f)=1\right]=$ $S^{(q)}(f)$. The frequency occupancy probability for a random bandwidth demand is related to its CDF and the spectrum assignment scheme for each realization. Assuming that each realization $\delta$ for demand $q$ is centered at frequency $f_{q}$,

$$
S^{(q)}(f)=1-P_{\Delta}^{(q)}\left(2\left|f-f_{q}\right|\right)
$$


We consider two representative traffic models as examples: uniform and truncated normal distributed random bandwidth traffic. A uniform distribution is often used when one does not have information about the traffic other than the minimum and maximum bandwidth. For bandwidth uniformly distributed over $[60,140] \mathrm{GHz}$ and centered at $f_{q}=200 \mathrm{GHz}$, $S^{(q)}(f)$ calculated using (1) is shown in Fig. 1 (a). When the traffic bandwidth is known to have a given mean and variance, it is often better to model the bandwidth as having a truncated normal distribution. For our results, we truncate the lower end of the distribution at three sigma or $30 \mathrm{GHz}$, whichever is larger. $S^{(q)}(f)$ for normal distributed traffic with the same mean and variance as the aforementioned uniform distribution is shown in Fig. 1 (b).

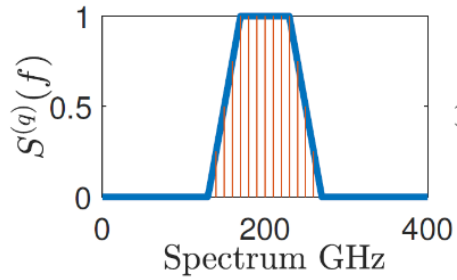

(a)

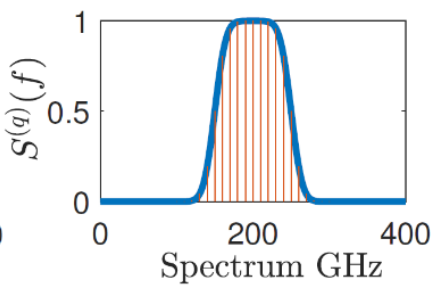

(b)
Fig. 1 Occupancy probability of (a) uniformly distributed and (b) normal distributed traffic bandwidth

\section{Probabilistic Spectrum Gaussian Noise Model}

The PSGN calculates the expected value of the noise experienced by a random-bandwidth signal of interest caused by itself and other signals on the same link that also have a random bandwidth, given the traffic model. In this section, we derive the analytical expressions for the expected noise.

By using the GN model approximation in [3, eq. (16)], the PLI power spectral density (PSD) $G_{N}$ per span per polarization becomes

$$
\begin{aligned}
G_{N} & =G_{A S E}+G_{N L I}, \\
G_{N L I} & =G_{S C I}+\sum_{q} G_{X C I, q},
\end{aligned}
$$

where, $G_{A S E}, G_{N L I}$ and $G_{S C I}$ represent the PSD of the amplified spontaneous emission (ASE), nonlinear interference (NLI) and self-channel interference (SCI), respectively, and $G_{X C I, q}$ represents the PSD of the cross channel interference (XCI) contributed by channel $q$. For a signal of interest centered at frequency 0 ,

$$
\begin{gathered}
G_{S C I}=\mu G^{3} \ln \left(\rho \Delta^{2}\right), \quad(4) \\
G_{X C I, q}=\mu G G_{q}^{2} \ln \left(\frac{f_{q}+\Delta_{q} / 2}{f_{q}-\Delta_{q} / 2}\right) .
\end{gathered}
$$

$\rho=\frac{\pi^{2}\left|\beta_{2}\right|}{\alpha}, \mu=\frac{3 \gamma^{2}}{2 \pi \alpha\left|\beta_{2}\right|}, \alpha$ is the fiber loss parameter, $\gamma$ represents the fiber nonlinearity parameter, and $\beta_{2}$ represents the group velocity dispersion parameter. $\Delta$ and $G$ represent the bandwidth and signal power spectral density for the channel of interest, the random variable $\Delta_{q}$ represents the bandwidth of channel $q$, and $G_{q}$ is the PSD of channel $q$. We assume that $G=G_{q}[4]$. The ASE noise only depends on the transmission length, and thus we focus on estimating the nonlinear interference (SCI and XCI) for random bandwidth demands.

The SCI for the channel of interest depends primarily on its own bandwidth. The expected SCI noise becomes

$$
\begin{aligned}
& E\left[G_{S C I}\right]=\mu G^{3} E\left[\ln \left(\rho \Delta^{2}\right)\right] \\
= & \mu G^{3} \int_{-\infty}^{\infty} \ln \left(\rho \delta^{2}\right) f_{\Delta}^{(q)}(\delta) d \delta,
\end{aligned}
$$

and its variance can be given as

$$
\operatorname{Var}\left[G_{S C I}\right]=\mu^{2} G^{6} \int_{-\infty}^{\infty} \ln ^{2}\left(\rho \delta^{2}\right) f_{\Delta}^{(q)}(\delta) d \delta-E^{2}\left[G_{S C I}\right] .
$$

These integrals can be solved exactly for a uniformly distributed bandwidth demand, but in general must be computed numerically.

The XCI for the channel of interest depends on the center frequency difference between itself and the interfering channel, and its bandwidth, which in this paper is a random variable with a given distribution. Using (5), we can write the XCI assuming $\Delta_{q}$ is a random bandwidth with realization $\delta \in$ $\left[\delta_{\text {min }}, \delta_{\text {max }}\right]$. Without violating the assumptions given in [3, eq. (16)], the XCI contributed by demand $q$ with bandwidth $\Delta_{q}$ equals the sum over frequency differentials $d f$ where $\sum d f=$ $\Delta_{q}:$

$$
E\left[G_{X C I, q}\right]=\mu G^{3} E\left[\sum_{i=-\Delta_{q} / 2 d f}^{\Delta_{q} / 2 d f} \ln \left(\frac{f_{q}+i d f+d f / 2}{f_{q}+i d f-d f / 2}\right)\right] .
$$

Using the indicator function $I^{(q)}(f)$, we write the expected noise due to signal $q$ as

$E\left[G_{X C I, q}\right]$

$$
\begin{aligned}
& =\mu G^{3} E\left[\sum_{i=-\infty}^{\infty} I^{(q)}\left(f_{q}+i d f\right) \ln \left(\frac{f_{q}+i d f+d f / 2}{f_{q}+i d f-d f / 2}\right)\right] \\
& =\mu G^{3} \sum_{i=-\infty}^{\infty} S^{(q)}\left(f_{q}+i d f\right) \ln \left(\frac{f_{q}+i d f+d f / 2}{f_{q}+i d f-d f / 2}\right),
\end{aligned}
$$

because $I^{(q)}\left(f_{q}+i d f\right)$ is the only random quantity in (10). We can thus conclude that the expected XCI can be written as a Riemann sum of the product of each noise component's strength times its presence probability over the entire spectrum. When $d f \rightarrow 0$, the discrete sum becomes an integral. Using the fact that when $d x \rightarrow 0, \ln \frac{x+d x / 2}{x-d x / 2}=\frac{1}{x} d x$, (10) can be further simplified as

$$
E\left[G_{X C I, q}\right]=\mu G^{3} \int_{-\infty}^{\infty} \frac{1}{f} S^{(q)}(f) d f .
$$

According to the nature of the nonlinearity, the SCI noise is stronger than the XCI noise. Furthermore, since the XCI is typically made up of many channels, its variance is expected to be low compared to that of the SCI (usually more than 10 times lower), as we have empirically observed through simulation. We hence assume the variance of the XCI noise can be neglected.

The PSGN model uses the variance and expected value of SCI noise and the expected value of the XCI noise to provide a conservative estimate of the NLI noise. The PSGN PSD can be written as 


$$
G_{P S G N}=E\left[G_{S C I}\right]+r \sqrt{\operatorname{Var}\left[G_{S C I}\right]}+\sum_{q} E\left[G_{X C I, q}\right],
$$

where $r$ is a variable defining how conservative the estimate is. When $r=0$, the PSGN estimates the expected value of the nonlinear interference.

\section{Numerical Results and Validation}

In this section, we show numerical results for the two traffic models considered in this work, the uniform and (truncated) normal bandwidth models. We then validate the proposed PSGN model using Monte Carlo simulations.

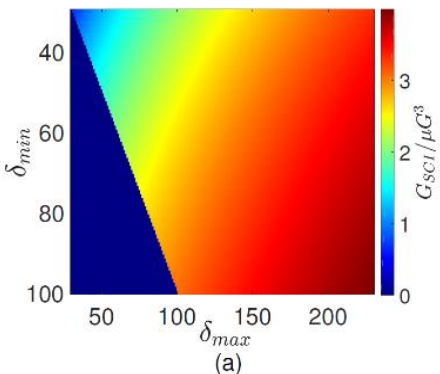

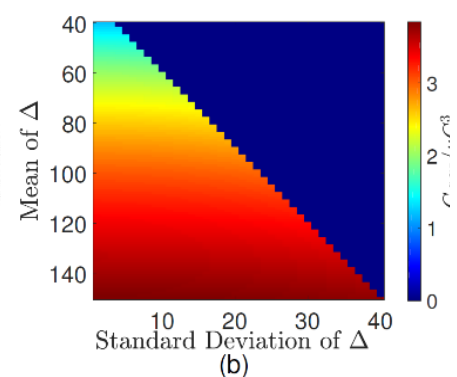

(b)
Fig. 2 PSGN estimated SCI of (a) uniformly distributed and (b) normal distributed random bandwidth demand.

In Fig. 2 (a) we show the SCI noise for a uniformly distributed random bandwidth demand for typical values of the minimum and maximum bandwidth. The PSGN estimated SCI is from 0.7 to 4.3 times $\mu G^{3}$. In Fig. 2 (b), we show the SCI for a normally distributed bandwidth demand as a function of the mean and variance of the bandwidth. The PSGN estimated SCI is from 1.3 to 3.8 times $\mu G^{3}$.

We then validate the proposed PSGN model by using Monte Carlo simulations. Demands are randomly generated according to their distributions for 1,000,000 Monte Carlo trials. The normalized error of the PSGN with $r=0$ is found to be in the order of $10^{-3}$ compared to the mean value NLI of the randomly simulated demands.

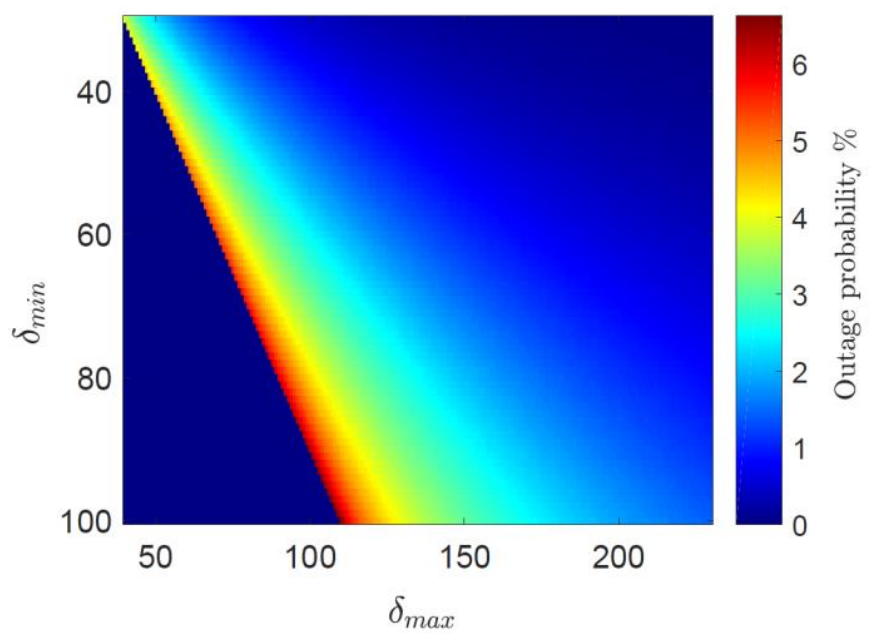

Fig. 3 Outage probability for uniformly distributed bandwidth demands with various $\left[\delta_{\min }, \delta_{\max }\right]$. It shows the probability that the NLI from the Monte Carlo simulation exceeds the NLI estimated by the PSGN for $r=2$.
Fig. 3 shows through simulation that the PSGN model provides a conservative estimate of the PLIs using the traffic models described in Section 2. The channel of interest is centered at $0 \mathrm{GHz}$. Ten demands share the link with the channel of interest, with $10 \mathrm{GHz}$ guard-bands between them. 1,000,000 Monte Carlo trials are conducted to test the outage probability of the PSGN, i.e., the probability that the simulated NLI exceeds the PSGN model. The results show that for the PSGN with $r=2$, the average outage probability is $1.4 \%$ for the cases tested.

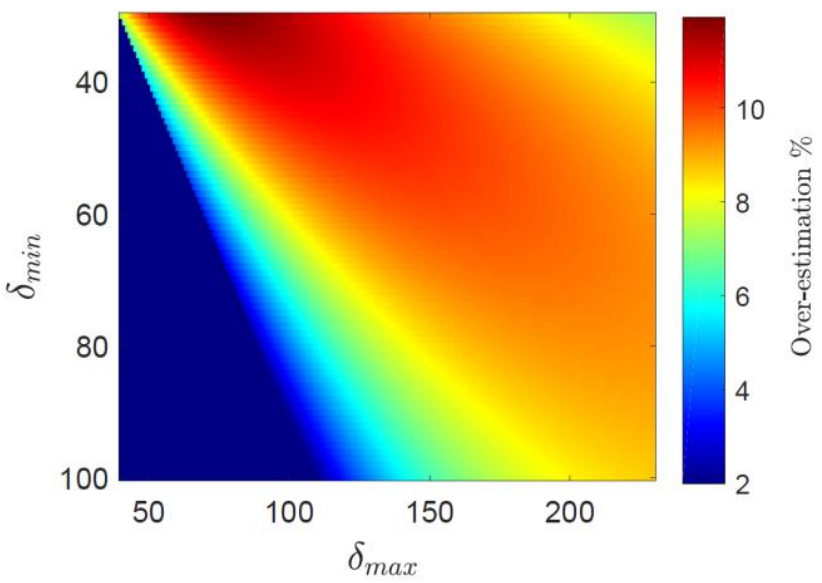

Fig. 4 Percent of NLI over-estimated by the maximum bandwidth GN compared to the PSGN ( $r=2)$ for uniformly distributed bandwidth.

Fig. 4 shows the ability of the PSGN to lower the expected NLI compared with the maximum bandwidth GN for the same distributions as in Fig. 3. The amount that the maximum bandwidth GN over-estimates the NLI compared with the PSGN is measured by $\frac{\max \left(G_{N L I}\right)-G_{P S G N}}{G_{P S G N}}$, where $\max \left(G_{N L I}\right)$ represents the NLI estimated by the maximum bandwidth GN. The maximum bandwidth estimates the worst case NLI noise and has zero outage probability. However, it results in an average of $9.1 \%$ and as high as $14 \%$ over-estimated NLI compared to the PSGN, depending on the distribution's parameters, wasting network resources most of the time.

\section{Conclusion and Acknowledgements}

The proposed PSGN model calculates the expected nonlinear noise for random bandwidth traffic based on the GN model given the probability distribution of the bandwidth. We derive closed-form expressions for the noise mean and variance. It provides a more accurate performance estimate than using the maximum bandwidth GN model typically used in standard provisioning. Most importantly, the proposed PSGN algorithm yields a simple mathematic expression that is time-efficient to compute. The PSGN algorithm can accommodate both offline and online resource allocation algorithms.

On average, the PSGN model experiences a $1.4 \%$ noise outage while gaining $9.1 \%$ in lower NLI estimates compared to the maximum bandwidth GN model.

This work was supported in part by NSF grant CNS-1718130. 


\section{References}

[1] O. Gerstel, M. Jinno, A. Lord, and S. Yoo, "Elastic optical networking: A new dawn for the optical layer?" IEEE Commun. Mag., vol. 50, no. 2, pp. 12-20, 2012.

[2] P. Poggiolini, G. Bosco, A. Carena, V. Curri, Y. Jiang, and F. Forghieri, "The GN-model of fiber non-linear propagation and its applications," J. Lightwave Technol., vol. 32, no. 4, pp. 694-721, 2014.

[3] P. Johannisson and E. Agrell, "Modeling of Nonlinear Signal Distortion in Fiber-Optic Networks," J. Lightwave Technol., vol. 32, no. 23, pp. 4544-4552, 2014.

[4] Y. Xu, L. Yan, E. Agrell and M. Brandt-Pearce, "Iterative resource allocation algorithm for EONs based on a linearized GN model," J. Opt. Commun. Netw., vol. 11, no. 3, pp. 39-51, 2019.

[5] L. Yan, E. Agrell, H. Wymeersch, and M. BrandtPearce, "Resource allocation for flexible-grid optical networks with nonlinear channel model," J. Opt. Commun. Netw., vol. 7, no. 11, pp. B101-B108, 2015.

[6] L. Yan, Y. Xu, M. Brandt-Pearce, N. Dharmaweera, and E. Agrell, "Robust regenerator allocation in nonlinear flexiblegrid optical networks with time-varying data rates," J. Opt. Commun. Netw., pp. 823-831, 2018.

[7] V. Curri, M. Cantono, and R. Gaudino, "Elastic all-optical networks: a new paradigm enabled by the physical layer. How to optimize network performances?" J. Lightwave Technol., vol. 35, no. 6, pp. 1211-1221, 2017. 